\title{
Constraints on Strategy Construction in a Speeded Discrimination Task
}

\author{
Gordon D. Logan \\ Erindale College, University of Toronto \\ Mississauga, Ontario, Canada
}

\author{
N. Jane Zbrodoff \\ Ontario Institute for Studies in Education \\ University of Toronto \\ Toronto, Ontario, Canada
}

\begin{abstract}
In three experiments, subjects reported the identity of a word (ABOVE or BELOW) that appeared above or below a fixation point. On some trials, a cue presented $100,200,400,600,800$, or 1,000 msec before the word indicated the relation between position and identity (i.e., whether the dimensions were compatible, e.g., ABOVE/above and BELOW/below, or conflicted, e.g., ABOVE/below and BELOW/ above). On the other trials, the cue was withheld (Experiment 2) or it bore no information about the relation between dimensions (Experiments 1 and 3 ). In each experiment, the cue reduced reaction time below the level of no-cue or neutral-cue controls, indicating strategic use of the relation between dimensions. Experiments 1 and 2 manipulated the number of potential cues that could occur in a block. A stronger cuing effect was found when one cue could occur (Experiment 2) than when two cues could occur (Experiment 1). Experiment 3 manipulated practice; it revealed that with practice the cuing effect reached asymptote at shorter delays. The asymptote itself did not change. Experiment 4 showed that cue-delay effects were independent of warning interval (warning interval and cue delay were confounded in Experiments 1,2, and 3). The experiments demonstrate construction and utilization of strategies; they show that construction is sensitive to constraints imposed by the subject's goals and abilities and by the structure of the task environment.
\end{abstract}

This article reports an investigation of strategies for performing a speeded discrimination task. It is based on the premise that nearly all cognitive tasks are performed as strategies, and that cognitive psychology can advance only by coming to understand what strategies are and how they are constructed and utilized. In general, a strategy may be defined as an optional organization of cognitive processes designed to achieve some

The order of authorship was determined randomly.

This research was supported by Grant No. A0682 to Gordon Logan from the Natural Sciences and Engineering Research Council of Canada. Jane Zbrodoff was supported by a doctoral fellowship from the Social Sciences and Humanities Research Council of Canada.

We are grateful to Kathy Constantinou and Ken Davis for testing the subjects, and to Frank Restle, Margaret Jean Intons-Peterson, Jonathan Baron, Margaret Schadler, and Tom Carr for valuable comments on the article.

Requests for reprints should be sent to Gordon D. Logan, Department of Psychology, Erindale College, University of Toronto, Mississauga, Ontario, Canada L5L 1 C6. goals in some task environment. The strategy chosen is probably a compromise between the constraints imposed by the informational structures in the task environment, by the resources, capacities, abilities, or processes in the subject's cognitive repertoire, and by the structure of the goals to be obtained by performing. This suggests that strategies may be investigated experimentally by manipulating the different constraints. This approach was adopted in the experiments reported here.

The task we studied required subjects to report the identity of a briefly presented word (either ABOVE or BELOW). The primary goals, defined by the instructions, were to respond as quickly and accurately as possible. This one-dimensional discrimination task was complicated by introducing variation in another dimension, the position the word occupied on the screen: The word appeared above or below the fixation point. In order to achieve the goal of responding accurately, subjects would have to attend se- 
lectively to the word's identity and try to filter out its position.

However, if the dimensions were correlated and subjects knew the relation between dimensions, two more options would be available to achieve the accuracy goal: Subjects could attend selectively to the unreported dimension (i.e., position) and report the related value of the reported dimension (e.g., if ABOVE always appeared below the fixation point and BELOW always appeared above the fixation point, they could set themselves to discriminate the word's position, and say "above" whenever they saw something below the fixation point and "below" whenever they saw something above the fixation point). Alternatively, they could divide attention between dimensions, basing their response partly on the word's position and partly on its identity (e.g., by responding to a weighted combination of information from the two dimensions; see Logan, 1980; Logan \& Zbrodoff, 1979). If the display conditions were such that position could be discriminated faster than identity, these two strategies could increase the speed of responding without sacrificing accuracy and better achieve the primary goals of performing.

An obvious way to study these strategies is to inform subjects about the relation between dimensions. This can be done in (at least) two ways: First, the relative frequency of different kinds of trials can be manipulated. In our previous research, we varied the relative frequency of compatible (e.g., ABOVE/above and BELOW/below) and conflicting (e.g., ABOVE/below and BELOW/ above) trials so that subjects could predict the value of the reported dimension (identity) once they knew the value of the unreported dimension (position). (For example, if conflicting trials were more frequent, anything below the fixation point was likely to be the word ABOVE, and anything above the fixation point was likely to be the word BELow.) We found that subjects picked up the correlation between dimensions and used it to speed their responses. Their performance suggested that they adopted a divided-attention strategy, attending to the unreported dimension in proportion to its validity as a cue to the value of the reported dimension (Logan \& Zbrodoff, 1979; see also Greenwald \& Rosenberg, 1978; Logan, 1980).
The earlier studies focused on how subjects utilized the correlation between dimensions and said very little about how they acquired the correlation. Generally, the strategies require that subjects construct some representation of the relation between dimensions, which they then utilize in applying the strategy. The present article is primarily concerned with the construction process and with distinguishing it from the utilization process. The experiments exploited a second method of informing subjects about the relation between dimensions: Before each trial, subjects were presented with a cue (an X or an O) that indicated whether the dimensions of the next stimulus would be compatible or conflicting (i.e., $\mathrm{X}$ always preceded ABOVE/above or BELOW/ below; $O$ always preceded $A B O V E /$ below or BELOw/above). By varying the delay between the onset of the cue and the onset of the word, we traced the time-course of construction. Since construction must precede utilization, the time-course function should appear as an approach to an asymptote as delay increases. The approach to asymptote should be gradual because the time required for construction should vary somewhat, and at the earlier delays, the word might appear before construction is complete. The time taken to reach asymptote is a measure of the latency of the construction process (i.e., the time required for the probability of completing construction to equal 1); the difference between asymptotic performance and performance in control trials is a measure of the effectiveness of utilization.

We used two kinds of control trials to assess the cuing effect in the present experiments: Experiments 1, 3, and 4 used neutralcue controls. An X or an $\mathrm{O}$ was presented on each trial, but the cue carried no information about the relation between the dimensions of the following stimulus word (i.e., $\mathrm{X}$ preceded compatible stimuli as often as it preceded conflicting stimuli; likewise for $0)$. These neutral trials would produce the same general alerting effect as the valid cues (see Posner \& Boies, 1971) but would not provide information that would allow subjects to adopt strategies of dividing attention or attending selectively to the unreported dimension. Experiment 2 used no-cue control trials, in which the cue was withheld. 
Note that the utilization process has to work with what it was given by the construction process, so the level of asymptotic performance may also indicate limitations on the construction process. In particular, it is possible that subjects will engage the construction process probabilistically, responding to the word's position in the manner dictated by the cue on some proportion of the trials, and responding to the word's identity on the remaining trials, ignoring the cue. This would produce mean cued reaction times that fell below the no-cue reaction times to word identity, but above no-cue reaction times for judgments about the word's position, mimicking the effects of an inefficient utilization process. Fortunately, probabilistic construction has implications for the variance of cued reaction times that are not shared by the inefficient utilization hypothesis: Probabilistic construction would inflate the variance of cued reaction times, relative to neutral-cue or no-cue controls. The variance would increase because cued reaction times would be based on two distributions with different means (i.e., the neutral-cue or no-cue word reaction time distribution and the neutral-cue or no-cue distribution of reaction times to judge the word's position), and the difference between the means would contribute to the variance. The difference between means would not affect the variance of control reaction times, which would be based on only one distribution (i.e., the neutral-cue or no-cue word reaction time distribution).

By contrast, there is no reason to expect cued standard deviations to be larger than no-cue or neutral-cue controls under the inefficient utilization hypothesis. Under that hypothesis, cued reaction times would be based on one distribution reflecting a weighted combination of information about the identity and the position of the word; the difference between the means of the no-cue or neutral-cue reaction times to the word's position and identity would not contribute to the variance. Further, under the inefficient utilization hypothesis, cued variance may even be smaller than no-cue or neutralcue variance if cued reaction times are faster than neutral-cue or no-cue controls, because reaction time variance generally increases with the mean. (Indeed, this is a general property of random-walk models, which provide good fits to reaction time data in a variety of contexts, e.g., Ratcliff, 1978.)

The stimuli we used also allowed us to investigate Stroop-type facilitation and interference. Generally, when the meaning of an unreported dimension conflicts with the meaning of the reported dimension, the time to report the value of the reported dimension increases relative to controls in which the unreported dimension is neutral. When the meanings of the reported and unreported dimensions are compatible, the reported dimension is reported faster than controls in which the unreported dimension is neutral (for reviews, see Dyer, 1973; Jensen \& Rohwer, 1966; Logan, 1980). Thus, in the present experiments, conflicting stimuli (ABOVE/ below and BELOW/above) should take longer to process than compatible stimuli (ABOVE) above and BELOw/below). Ideally, Strooplike facilitation and inhibition should be assessed against controls in which the position of the word is neutral, as would be the case if the words appeared in the center of the screen. However, because of the acuity gradient on the retinae, words presented centrally are likely to be processed faster than words presented peripherally, so the "neutral" controls may be faster than both compatible and conflicting trials. Indeed, this is what Palef (1978) found when she included such "controls" in her design. It would be possible to estimate the acuity effect by having subjects identify words that did not specify positions (e.g., APPLE, PEACH), presented centrally and peripherally. The acuity effect could then be subtracted from reaction times to the Stroop-type stimuli to assess facilitation and interference. This was far too cumbersome for our purposes, since we were more interested in strategies than in the Stroop effect per se. We assessed the Strooplike effect from the difference in reaction time to compatible and conflicting stimuli, which should represent the sum of the facilitation and interference effects.

\section{Experiments 1 and 2}

The first two experiments together address the effects of an informational constraint on the time-course of construction. In Experiment 1 , the $X$ and the $O$ were both valid in 
the same blocks, so subjects had to detect the cue and discriminate $X$ from $O$ in order to decide what to construct. This is analogous to a choice reaction time task. In Experiment 2, however, the $X$ and the $O$ were presented (and were valid) in different sessions, so subjects only had to detect the cue to determine what to construct (to ensure that the occurrence of the cue actually provided information, it was withheld on $66 \%$ of the trials). This is analogous to a simple reaction time task (with $66 \%$ catch trials). Many studies have shown that choice reaction time tasks are harder than simple reaction time tasks, so we might expect construction to be more difficult in Experiment 1 than in Experiment 2. The difference in difficulty could appear as a difference in the latency or the probability of construction, or both.

Since the two experiments were very similar, their methods are described in a single section.

\section{Method}

Subjects. Twenty-four second- and third-year undergraduates were paid for serving in two sessions. Twelve different subjects served in each experiment.

Apparatus and stimuli. The stimuli were the words ABOVE and BELOW, written in capital letters and presented above and below a central fixation point; and the cues $\mathrm{X}$ and $\mathrm{O}$, also written in capitals but presented in the center of the screen. The stimuli were displayed on a point-plot cathode ray tube (Tektronix Model 604, equipped with P31 phosphor) under the control of a PDP-11/03 laboratory computer. Each letter was formed by illuminating about 20 points in a $5 \times 7$ dot matrix, and subtended about $.43 \times .57$ degrees of visual angle when viewed at a distance of $60 \mathrm{~cm}$ (constant viewing distance was maintained by a headrest). Each word subtended about $2.68^{\circ}$ horizontally and $.57^{\circ}$ vertically, and appeared $2.77^{\circ}$ above or below the fixation point. The display program "painted" one dot on the screen every $78 \mu \mathrm{sec}$; the brightness of the single-letter cues was made the same as the five-letter words by painting four extra letters at a nonexistent position on the screen before refreshing the cue.

Each trial began with a $1000-\mathrm{Hz}$ warning tone and a fixation point. The tone sounded for $500 \mathrm{msec}$ and was followed by a 500 -msec silent interval before the word appeared. The word was exposed for $500 \mathrm{msec}$ and was followed by a $2.5-\mathrm{sec}$ intertrial interval. The cue, when it appeared, was exposed for $100 \mathrm{msec}$; its onset preceded the onset of the word by one of six delays: 100 , $200,400,600,800$, or 1,000 msec. Since the delay between the warning tone and the word was fixed, the delay between the warning tone and the cue varied inversely with the delay between the cue and the word (i.e., the two delays summed to $1,000 \mathrm{msec}$ ). Experiment
4 demonstrated that the confound between cue delay and warning interval was not important.

Subjects indicated which word had appeared by pressing the rightmost or the leftmost of a panel of eight response buttons. The computer recorded which button was pressed and the reaction time elapsing between the onset of the word and the onset of the response.

Procedure. Subjects in both experiments were told that their task was to identify the words ABOVE and BELOW by pressing the appropriate button as quickly as possible while maintaining an error rate less than $10 \%$. Every subject pressed the right button for ABOVE and the left button for BELOW. Subjects were told that the position of the word on the screen was irrelevant. When the cue was valid, they were told that $X$ meant that ABOVE would always appear above the fixation point and BELOW below, and that $O$ meant that $A B O V E$ would always appear below the fixation point and BELow above. (Subjects in Experiment 2 were only told about the cue that was valid for that session.) They were told that pilot studies had shown that people could respond to the word's position faster than they could respond to the word itself, so the cues could help them to respond faster. They were told to pay careful attention to the cue and to try to use it on every trial because we were interested in how people used cues. This emphasis was necessary because it was difficult to use the cue and because pilot subjects who were not given special instructions had tended to ignore the cue. In Experiment 1 , in the session in which the cue was neutral, subjects were told that the cue would appear but that it bore no relation to the word, so they should ignore it.

1. Experiment 1 . In each session, each subject completed six blocks of 80 trials, one block for each cue delay. Within each block, the four possible combinations of words and positions (i.e., ABOVE/above, ABOVE/below, BELOw/above, and BELOw/below) occurred equally often. Thus, compatible and conflicting stimuli were equally frequent, as were the two responses.

The order of cue delays varied between subjects according to a balanced $6 \times 6$ Latin square, with two subjects assigned to each row. Each subject received the cue delays in the second session in the same order as he or she had in the first. Half of the subjects had the cue valid for the first session and neutral for the second, and the other half had the opposite. Assignment to these orders was orthogonal to the assignment to orders of cue delays. Each subject received the stimuli in a different random order each session.

2. Experiment 2. In each session, each subject completed six blocks of 96 trials, one for each cue delay. In each block, a cue was presented on $32(33 \%)$ trials and withheld on $64(66 \%)$. Half of the 64 no-cue trials involved compatible stimuli, and half involved conflicting stimuli. For one session, the cue was an $X$, which was always followed by a compatible stimulus; for the other session, the cue was an $O$, which was always followed by a conflicting stimulus. Thus, a single cue was valid each day, and the absence of the cue carried no information about the relation between dimensions. Note that in this design compatible stimuli are more frequent when $X$ is the cue, and conflicting stimuli are more frequent when $O$ is the cue. Nevertheless, the two responses occurred equally often.

As in Experiment 1, the order of cue delays was determined by a balanced $6 \times 6$ Latin square, with two 
subjects assigned to each row. One subject assigned to each row had the session with the $X$ cue before the session with the $O$ cue, and the other had the opposite. As in Experiment 1, each subject received the stimuli in a different random order each session.

\section{Results}

Experiment 1. Each subject completed 40 trials in each combination of the two compatibility conditions, the two cue validity conditions, and the six cue delays. Error rates and the mean reaction times and standard deviations for correct responses were calculated for each subject in each condition. The mean reaction times across subjects are displayed in Figure 1 as a function of cue delay. Each point in the figure is based on a maximum of 480 observations.

Three aspects of the data in the figure are relevant to our present concerns. The first is the obvious cue validity effect: The cue reduced reaction time below neutral-cue controls, at least with compatible stimuli at the longer cue delays. This demonstrates strategic use of the relation between dimensions, and the interaction with cue delay describes its time-course. Second, reaction time was longer when the dimensions of the stimulus conflicted than when they were compatible, indicating a Stroop-like effect. Third, the cue validity effect was considerably stronger with compatible stimuli than with conflicting stimuli. Put another way, the compatibility effect was stronger when the cue was valid than when it was neutral.

Each of these effects was apparent in a $2 \times 2 \times 6$ analysis of variance (ANOVA) performed on the reaction time data. First, although the main effect of cue validity was not significant, $F(1,11)=1.81, M S_{e}=$ $22,057.37$, the main effect of delay was highly significant, $F(5,55)=10.26, p<.01$, $M S_{\mathrm{e}}=1,971.70$, as was the interaction between cue validity and delay, $F(5,55)=$ $7.40, p<.01, M S_{\mathrm{e}}=1,162.23$, indicating that reaction times to stimuli following valid cues were faster than reaction times to stimuli following neutral cues, at the longer cue delays. Second, when the cues were neutral, reaction time was longer with conflicting stimuli than with compatible ones, $F(1$, $11)=14.07, p<.01, M S_{\mathrm{e}}=1,301.54$. Overall, the main effect of compatibility was significant as well, $F(1,11)=37.15, p<$

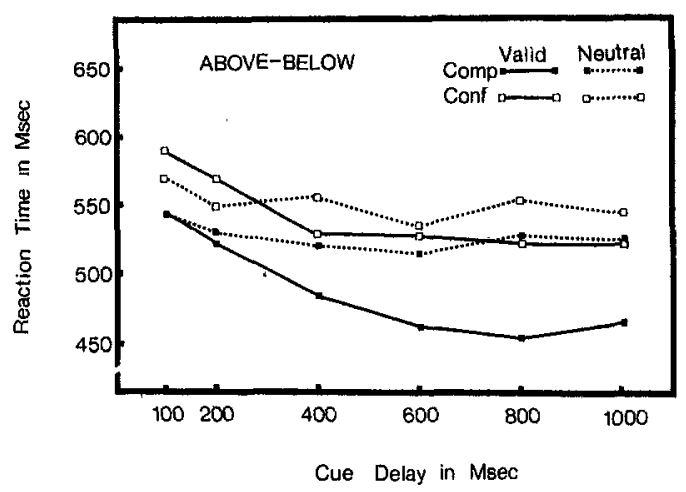

Figure 1. Mean reaction time as a function of cue delay in Experiment 1. (Compatibility and cue validity are parameters. Comp $=$ compatible trials; Conf $=$ conflicting trials; Valid $=$ perfect cue validity; Neutral $=$ no cue validity.)

$.01, M S_{\mathrm{e}}=2,819.18$. Third, the interaction between cue validity and compatibility was also significant, $F(1,11)=13.43, p<.01$, $M S_{\mathrm{e}}=1,301.54$, indicating that the cue validity effect was stronger with compatible stimuli than with conflicting stimuli.

The mean standard deviations across subjects are displayed in Table 1 . The important result here was that the standard deviations of the cued reaction times were larger than the standard deviations of the neutral-cue control reaction times, $F(1,11)=7.08, p<$ $.05, M S_{\mathrm{e}}=5,598.38$, which suggested that subjects were switching attention between dimensions rather than dividing attention. No other effects were significant.

The mean error rates across subjects also appear in Table 1. More errors occurred when the cue was valid than when it was neutral, reflecting (at least empirically) a speed-accuracy trade-off apparent in other studies of cuing (e.g., LaBerge, 1973). Also, more errors tended to occur with conflicting stimuli than with compatible ones, and this effect was more pronounced when the cue was valid.

Experiment 2. Each subject completed 32 trials in each combination of compatibility, cue validity, and cue delay conditions. Error rates and the means and standard deviations of the reaction times from correct responses were calculated for each subject for each condition. The mean reaction times across subjects are displayed as a function of cue delay in Figure 2. Each point in the 
Table 1

Mean Proportions of Error and Standard Deviations of Reaction Time in Experiment 1 as a Function of Cue Validity, Compatibility, and Cue Delay

\begin{tabular}{ccccccc}
\hline & \multicolumn{6}{c}{ Cue delay (msec) } \\
\cline { 2 - 7 } Trial & 100 & 200 & 400 & 600 & 800 & 1,000 \\
\hline \multicolumn{6}{c}{ Cue valid } \\
Comp & & \multicolumn{1}{c}{} & & & \\
Error & .095 & .093 & .068 & .060 & .055 & .053 \\
$S D$ & 119 & 114 & 128 & 124 & 127 & 128 \\
Conf & & & & & & \\
Error & .138 & .130 & .143 & .113 & .165 & .140 \\
$S D$ & 129 & 138 & 132 & 116 & 129 & 126 \\
\hline
\end{tabular}

Cue neutral

\begin{tabular}{crrrrrr}
$\begin{array}{c}\text { Comp } \\
\text { Error }\end{array}$ & .042 & .048 & .056 & .075 & .043 & .102 \\
$S D$ & 106 & 101 & 100 & 96 & 114 & 105 \\
Conf & & & & & & \\
Error & .069 & .042 & .071 & .073 & .077 & .056 \\
$S D$ & 103 & 101 & 88 & 109 & 96 & 109 \\
\hline
\end{tabular}

Note. Comp $=$ compatible; Conf $=$ conflicting.

figure is based on a maximum of $384 \mathrm{ob}-$ servations.

Three aspects of the data in the figure are relevant to our present concerns. First, there was a robust cue validity effect: Presenting the cue reduced reaction time substantially for both compatible and conflicting stimuli. As in Experiment 1, the cue validity effect was stronger at the longer delays. Second, reaction time was generally longer with conflicting stimuli than with compatible ones, replicating the Stroop-like effect seen earlier; there were some interesting interactions, however: In particular, the difference between compatible and conflicting reaction times in the no-cue trials depended on which cue was valid; the no-cue compatibility effect was very strong $(66 \mathrm{msec})$ in the context of the $X$ cue, which cued compatible stimuli, but actually reversed $(-7 \mathrm{msec})$ in the context of the $\mathbf{O}$ cue, which cued conflicting stimuli. Third, the cue validity effect was much stronger when compatible stimuli were cued than when conflicting stimuli were cued.

Each of these effects was confirmed in a 3 (condition, i.e., cue valid vs. no-cue control from compatible-cue trials vs. no-cue control from conflicting-cue trials) $\times 2$ (compatible vs. conflicting) $\times 6$ (cue delay) ANOVA performed on the reaction time data. First, the cue validity effect was apparent in the main effect of conditions, which was significant in the overall analysis that included data from cue-valid trials, $F(2,22)=49.43, p<.01$, $M S_{\mathrm{e}}=9,581.74$, but not in a contrast comparing data from the two no-cue conditions by themselves, $F(1,11)<1, M S_{e}=$ $10,442.76$. This indicates that cued reaction times were faster than no-cue controls, and that the no-cue controls did not differ from each other. Further, the main effect of delay was significant, $F(5,55)=6.61, p<.01$, $M S_{\mathrm{e}}=1,564.34$, and interacted strongly with conditions, $F(10,110)=13.61, p<.01$, $M S_{\mathrm{e}}=913.97$, in the overall analysis. Neither effect was significant in a contrast including only data from the no-cue conditions, $F_{\mathrm{s}}(5,55)<1, M S_{\mathrm{e}}=913.97$. This indicates that the advantage of cued reaction times over no-cue controls increased with cue delay.

Second, the Stroop-type effect was apparent as a main effect of compatibility in an analysis of the no-cue data by themselves; overall, no-cue conflicting reaction times were slower than no-cue compatiblè reaction

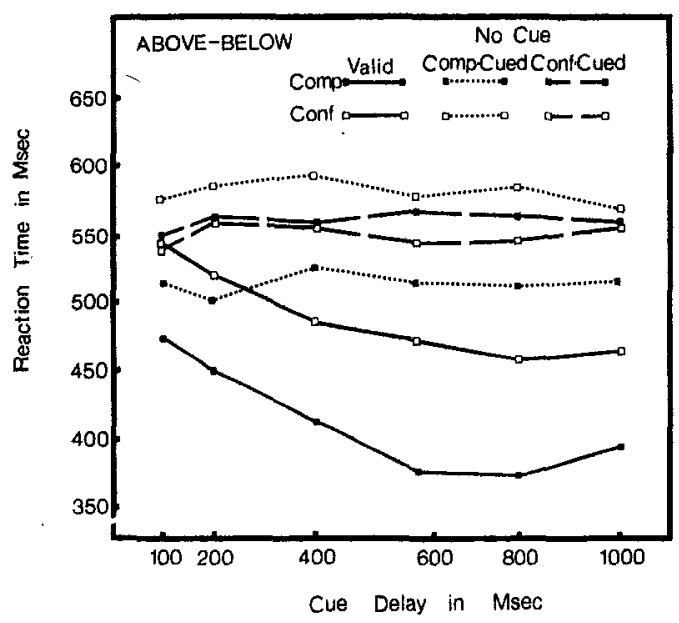

Figure 2. Mean reaction time as a function of cue delay in Experiment 2. (Note that the compatible, cue-valid data were obtained in the same session as the no-cue compatible-cued compatible and the no-cue compatible-cued conflicting data; similarly, the conflicting cuevalid data were obtained in the same session as the nocue conflicting-cued compatible and the no-cue conflicting-cued conflicting data.) 
times, $F(1,11)=26.02, p<.01, M S_{\mathrm{e}}=$ $2,263.3$. In this analysis, the interaction between compatibility and a factor representing the cue that was valid while the no-cue data were collected was highly significant, $F(1,11)=111.78, p<.01, M S_{\mathrm{e}}=958.86$, indicating that the compatibility effect was larger when $X$ was the cue that was valid (i.e., when compatible stimuli were cued) than when $O$ was the cue that was valid (i.e., when conflicting stimuli were cued).

Third, the finding that the cue validity effect was stronger with compatible stimuli than with conflicting stimuli was apparent in a contrast that compared the compatibility effect when the cue was valid with the average compatibility effect from the no-cue trials, $F(1,22)=26.90, p<.01, M S_{\mathrm{e}}=$ 2,026.36.

The mean standard deviations across subjects are presented in Table 2. Again, there was evidence that subjects switched attention between dimensions; cued standard deviations were larger than the no-cue standard deviations for conflicting stimuli. However, for compatible stimuli, cued standard deviations tended to be smaller than no-cue controls. A $3 \times 2 \times 6$ ANOVA was performed on the reaction time data (i.e., Cue Validity Conditions $\times$ Compatibility $X$ Delay). The only significant effect was the interaction between compatibility and conditions, $F(2,22)=6.98, p<.01, . M S_{\mathrm{e}}=$ 568.94. A contrast comparing conflicting cued standard deviations with the mean of the no-cue conflicting standard deviations was significant, $F(1,22)=9.90, p<.01$, $M S_{\mathrm{e}}=568.94$, indicating that cued standard deviations were larger than no-cue standard deviations. Another contrast comparing the compatible cued standard deviations with the mean of the no-cue compatible standard deviations was also significant, $F(1,22)=$ $4.59, p<.05, M S_{\mathrm{e}}=568.94$, indicating smaller standard deviations with the cue than without it.

The mean error rates across subjects also appear in Table 2. As in Experiment 1, subjects tended to be less accurate with the cue than without it. In the no-cue data, the pattern of errors reflected the interaction in the reaction times: Subjects were more accurate with compatible stimuli than with conflicting stimuli when $X$ was the cue. However,
Table 2

Mean Proportions of Error and Standard

Deviations of Reaction Time in Experiment 2

as a Function of Cuing Condition.

Compatibility, and Cue Delay

\begin{tabular}{lrrrrrr}
\hline & \multicolumn{6}{c}{ Cue delay (msec) } \\
\cline { 2 - 7 } Trial & 100 & 200 & 400 & 600 & 800 & 1,000 \\
\hline \multicolumn{7}{c}{ Cue valid } \\
Comp & & & & & & \\
$\quad$ Error & .050 & .059 & .053 & .031 & .034 & .016 \\
$S D$ & 104 & 76 & 73 & 96 & 93 & 95 \\
Conf & & & & & & \\
$\quad$ Error & .066 & .100 & .072 & .056 & .078 & .038 \\
$S D$ & 105 & 103 & 99 & 101 & 102 & 97 \\
\hline
\end{tabular}

No cue, compatible cue valid

\begin{tabular}{lrrrrrr} 
Comp & & & & & & \\
$\quad$ Error & .022 & .022 & .009 & .019 & .022 & .019 \\
$S D$ & 84 & 95 & 94 & 98 & 88 & 96 \\
Conf & & & & & & \\
$\quad$ Error & .113 & .069 & .069 & .065 & .078 & .075 \\
$S D$ & 83 & 90 & 83 & 83 & 90 & 91 \\
\hline
\end{tabular}

No cue, conflicting cue valid

\begin{tabular}{lrrrrrr} 
Comp & & & & & & \\
$\quad$ Error & .084 & .050 & .059 & .066 & .056 & .056 \\
$S D$ & 101 & 112 & 95 & 106 & 100 & 95 \\
Conf & & & & & & \\
$\quad$ Error & .047 & .022 & .038 & .022 & .025 & .025 \\
$S D$ & 84 & 91 & 91 & 106 & 102 & 91 \\
\hline
\end{tabular}

Note. Comp $=$ compatible; Conf $=$ conflicting.

they were more accurate with conflicting stimuli than with compatible stimuli when $O$ was the cue.

Experiments 1 and 2. It is clear in comparing Figures 1 and 2 that the cue validity effect was stronger in Experiment 2 than in Experiment 1. Moreover, it is clear that the difference was roughly uniform across the time-course function. To evaluate these differences statistically, we performed a $2 \times$ $2 \times 6 \times 2$ (Compatibility $\times$ Cue Validity $\times$ Cue Delay $\times$ Experiments) ANOVA on the reaction time data. ${ }^{1}$ In order to compare the

\footnotetext{
${ }^{1}$ We tried a more direct approach, fitting the cued reaction times to the equation$$
\text { RT cued }=c+a e^{-b(\text { cue delay), }}
$$

which represents an exponential approach to an asymptote. We planned ANOVAS on the parameter $c$, which
} 
no-cue data from Experiment 2 with the neutral-cue data from Experiment 1, we collapsed the two no-cue conditions, eliminating the effect of the cue that was valid while the no-cue data were collected. The interesting effects are the interactions with experiments: The only significant interaction was between experiments and cue validity, $F(1,22)=14.26, p<.01, M S_{\mathrm{e}}=14,305.06$, indicating a stronger cue validity effect in Experiment 2 than in Experiment 1. A contrast comparing the no-cue data with the neutral cue data found no significant difference, $F(1,22)<1, M S_{\mathrm{e}}=14,305.06$, indicating equivalent performance in the two experiments when subjects did not use the cues. Thus, the interaction reflects differences between the cue-valid conditions. A contrast comparing cue-valid data from the two experiments was highly significant, $F(1,22)=20.29, p<.01, M S_{\mathrm{e}}=14,305.06$. It is worth noting that the interaction between experiments, cue validity, and cue delay was not significant, $F(5,110)=1.74$, $p>.05, M S_{\mathrm{e}}=944.66$.

\section{Discussion}

There were three principal findings in Experiments 1 and 2: Foremost was the cue validity effect observed in both experiments. This replicates the strategic use of relations between dimensions seen in the earlier studies (Greenwald \& Rosenberg, 1978; Logan, 1980; Logan \& Zbrodoff, 1979). The second major finding was that the cue validity effect was much stronger in Experiment 2, in which only one cue was valid each block, than in Experiment 1, in which the two cues were valid in the same block. The major

represents the value of the asymptote, and the parameter $b$, which represents the rate of approach to the asymptote. Indeed, the equation fitted the means in Figures 1 and 2 quite well. In the individual data, however, the fit was significant in about half of the subjects, so we rejected the approach. Nevertheless, the parameter values for the average data agree with our conclusions: Reducing the number of cues changed the asymptote, $c$, from 454 to 374 for compatible stimuli and from 517 to 457 for conflicting stimuli, but it had little effect on the rate of approach to the asymptote, $b$. The values from Experiment 1 were .0039 and .0044 for compatible and conflicting stimuli, respectively, and the corresponding values from Experiment 2 were .0035 and .0036 . difference was in the level of the asymptote of the time-course function rather than in the rate of approach to asymptote, suggesting that the informational constraint, manipulated between experiments, affected the probability of construction or the efficiency of utilization rather than the speed of construction, as the analogy between simple and choice reaction time might suggest (i.e., simple reaction time is typically faster than choice reaction time, so construction should be faster in Experiment 2 than in Experiment 1 ). This suggests that the processes that follow detection and discrimination of the cue affect the speed of construction much more than do detection and discrimination themselves.

The standard deviation data suggest that the enhanced cue validity effect in Experiment 2 resulted from a higher probability of construction rather than more efficient utilization. The inflated cued standard deviations in both the compatible and the conflicting conditions of Experiment 1 and in the conflicting conditions in Experiment 2 suggest that subjects switched attention between dimensions, basing their responses on the word's position on some proportion of the trials and basing their responses on the word's identity on the remaining trials. The finding that compatible cued standard deviations were smaller than no-cue controls in Experiment 2 may seem contrary to this conolusion, but it need not be interpreted in that way: Cuing was so effective with compatible stimuli that the probabilistic construction hypothesis would predict that cued standard deviations would not be larger than no-cue controls; compatible cued reaction times were close to the $370 \mathrm{msec}$ necessary to report the word's position (Logan \& Zbrodoff, 1979, Experiment 1). Thus, subjects may have responded on the basis of the word's position on all of the trials, in which case cued standard deviations should equal the standard deviation of reaction times to judge the word's position. In our previous research, the standard deviations for position judgments were not different from standard deviations of word identity judgments (Logan \& Zbrodoff, 1979, Experiment 1); in the present Experiment 2, standard deviations from cued compatible trials should not be larger than no-cue controls. The fact that 
cued standard deviations were smaller than no-cue controls suggests that position standard deviations may have been smaller than no-cue-word standard deviations.

On the other hand, it is possible that subjects divided attention between dimensions on compatible trials in Experiment 2, basing their responses on a weighted combination of information from position and identity, yet switched attention in the other conditions in the two experiments. The data cannot distinguish between these alternatives.

The third major effect was that the cue validity effect was larger when compatible stimuli were cued than when conflicting stimuli were cued. Put another way, the compatibility effect was larger when the cue was valid than when the cue was neutral or withheld. This occurred in both experiments. We did not expect this effect, and will defer discussion of it until later. For now, we note that the effect in Experiment 2 was larger numerically (but not statistically) than the one in Experiment 1.

\section{Experiment 3}

The third experiment examined the effects of a constraint that the subject's ability places on construction and utilization. Its purpose was to provide a contrast with the effects of the informational constraint manipulated in Experiments 1 and 2. The task was the same as in Experiment 1 (i.e., the two cues were valid in the same block), but subjects performed in eight sessions instead of two. In the first and the eighth sessions, neutral-cue control data were gathered. In the second to the seventh sessions, subjects practiced with both cues perfectly valid. This experiment was designed to address two major questions. The first was whether practice would have the same effects as reducing the number of cues that were valid: Would practice enhance the cue validity effect uniformly across cue delay, producing a transition from the pattern of data in Experiment 1 to the pattern in Experiment 2? Or would practice affect the time-course function differently? The second question was whether practice would affect the asymmetry in cuing compatible versus conflicting stimuli. Would practice eliminate the advantage that compatible stimuli enjoy?

\section{Method}

Subjects. Six third-and fourth-year undergraduates from Erindale College of the University of Toronto were paid for serving in eight 1 -hour sessions. None had served in Experiments 1 or 2.

Apparatus and stimuli. These were the same as in Experiments 1 and 2.

Procedure. Each subject completed six blocks of $\mathbf{8 0}$ trials each day for 8 days. Cue validity was manipulated as in Experiment 1 (i.e,, the cue either had perfect validity or none at all). The cue was presented in each session but was valid only from Session 2 to Session 7, inclusive; Sessions 1 and 8 served as neutral-cue controls. Cue delay was varied and blocked as in Experiment 1 (i.e., one block each day for each of six delays: $100,200,400,600,800$, and $1,000 \mathrm{msec}$ ). On sessions in which the cue was valid, the order of cue delays was balanced within subjects according to a balanced $6 \times$ 6 Latin square. Each day, each subject received the cue delays in the order determined by a different row of the Latin square. The order in which subjects received the rows of the Latin square was balanced (between subjects) using another $6 \times 6$ Latin square. Each subject was assigned to one row of the second square, which determined the order in which he or she received the rows of the first. The order of cue delays in Session 1 was the same as the order in Session 7; the order in Session 8 was the same as the order on Session 2 (i.e., the orders in Sessions 1 and 8 continued the "cycle" determined by the Latin square for Sessions 2 through 7).

As in the previous experiments, each subject had $X$ as the cue for compatible stimuli and $O$ as the cue for conflicting stimuli, and each subject pressed the rightmost button for ABOVE and the leftmost for BELOW.

In the first and second sessions, the instructions were the same as in Experiment 1 (i.e., the instructions were given when subjects first experienced the neutral-cue and the cue-valid trials). In subsequent sessions, subjects were told whether or not the cue was valid. If it was valid, they were exhorted to do their best to use it. Each day they were reminded which button to press for which word.

\section{Results}

Each day, each subject completed 40 trials in each combination of the two compatibility conditions and the six cue delays. Error rates and the means and standard deviations of reaction times for correct responses were calculated for each subject. The mean reaction times across subjects are displayed in Figure 3. (Each point in the figure is based on a maximum of 240 observations.)

Three aspects of the data in the figure are relevant to our present concerns. First, there was an obvious cue validity effect for both compatible and conflicting stimuli, replicating the previous experiments. Second, the cue validity effect changed with practice; practice had its strongest effect on the time 


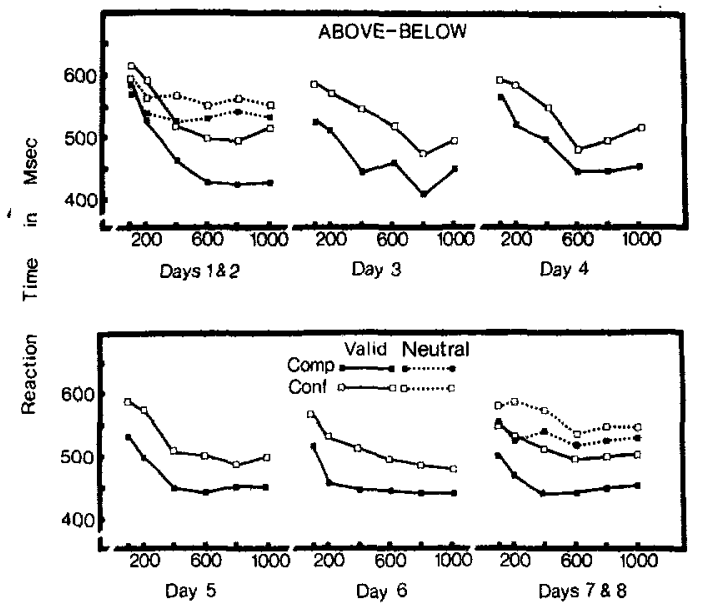

Figure 3. Mean reaction time as a function of cue delay in each session of Experiment 3. (Note that Days 1 and 8 involved neutral-cue control trials, and Days 2-7 involved cue-valid trials. Comp $=$ compatible trials; Conf $=$ conflicting trials.)

taken to reach asymptote (i.e., on the shorter cue delays) but it had very little effect on the asymptotic cued reaction times (i.e., at the longer cue delays). Third, it was clear that compatible stimuli benefited more from cuing than did conflicting stimuli and that the advantage persisted unchanged throughout practice.

Two ANOVAs were performed on the mean reaction times to assess the statistical support for these observations. One analysis included all the data (i.e., 2 compatibility conditions $\times 6$ delays $\times 8$ sessions), and one included only the data from Sessions 2 through 7 in which the cue was valid ( 2 compatibility conditions $\times 6$ delays $\times 6$ sessions). In general, the three points received statistical support: First, the effect of cue delay was significant in both analyses, $F \mathrm{~s}(5$, $25)=25.23$ and $21.27, p s<.01, M S_{\mathrm{e}} \mathrm{s}=$ $4,139.79$ and $5,291.32$, respectively. The interaction between cue delay and sessions was also significant in both analyses, $F(35,175)=$ 2.34, $p<.01, M S_{\mathrm{e}}=1,911.45$, and $F(25$, 125) $=1.79, p<.05, M S_{\mathrm{e}}=1,932.31$, respectively. The first interaction indicates the difference between cue-valid and neutral-cue reaction times (i.e., the cue validity effect): Fisher's least significant difference (LSD) test (Kirk, 1968) was used to compare cued reaction times from each session with the control values from corresponding delays from Sessions 1 and 8. It revealed that in every session, cued reaction times were faster than neutral-cue controls for delays of 400 msec or greater $(p<.05)$ except for the 400 msec delay in Session 4 with conflicting stimuli. The significant interaction in the analysis of the cue-valid data by themselves is relevant to the second point, that the time-course of cuing was affected by practice. From Figure 3 , it is apparent that practice improved performance at the early cue delays but had little effect on performance at the longer cue delays. To demonstrate this statistically, we assessed the simple main effects of sessions at each cue delay for compatible and conflicting trials. ${ }^{2}$ Each effect was based on 5 and 125 degrees of freedom, and the mean squared error was 494.25 . For compatible stimuli, the $F$ ratios from the $100-\mathrm{msec}$ to the $1,000-\mathrm{msec}$ delays, respectively, were $12.51, p<.01 ; 9.24, p<.01 ; 4.97, p<.01$; $1.45(n s) ; 4.50, p<.01$; and $1.43(n s)$. For conflicting stimuli, the $F$ ratios from the 100 msec to the 1,000 -msec delays, respectively, were $5.04, p<.01 ; 8.68, p<.01 ; 2.70, p<$ $.05 ; 1.39, n s ; 1.29, n s ;$ and $2.81, p<.05$.

The third point, that the cue validity effect was stronger with compatible stimuli and that this tendency remained constant throughout practice, was evident in several comparisons: The interaction between compatibility and sessions was significant in the overall analysis, $F(7,35)=2.59, p<.05$, $M S_{\mathrm{e}}=1,478.63$, but not in the analysis of the cue-valid data by themselves, $F(5,25)<$ $1, M S_{\mathrm{e}}=1,398.08$. The first interaction indicates that the compatibility effect was larger when the cue was valid than when it was neutral, and the second indicates that the compatibility effect remained constant

\footnotetext{
${ }^{2}$ We tried to fit an exponential equation to these data as well, and found that although the fit to the average data in Figure 3 was good, the fit to the individual data was significant in about half of the cases. However, the parameter values from the average data agree with our conclusions: Practice changed the rate of approach to the asymptote (at least for compatible stimuli) more than the asymptote itself. The values of the rate parameter, $b$, for Sessions $2-7$ were $.0050, .0040, .0037, .0112$, $.0069, .0184$, and .0118 for compatible stimuli, and $.0048, .0015, .0028, .0041, .0033$, and .0059 for conflicting stimuli. The values of the asymptote parameter, $c$, for Sessions 2-7 were 419, 428, 440, 447, 447, and 447 for compatible stimuli, and $496,438,491,488,476$, and 502 for conflicting stimuli.
} 
over the six sessions of practice with the cue valid.

The main effect of compatibility was significant in the overall analysis, $F(1,5)=$ $23.17, p<.01, M S_{\mathrm{e}}=14,890.77$, and a contrast comparing compatible and conflicting reaction times from neutral-cue trials from Sessions 1 and 8 was also significant, $F(1$, $35)=17.88, p<.01, M S_{\mathrm{e}}=1,478.63$, indicating a Stroop-like effect in the neutralcue trials.

The mean standard deviations across subjects are presented in Table 3 . The findings were somewhat unexpected. First, cued standard deviations remained relatively constant over Sessions 2-7. They were larger than the neutral-cue standard deviations from Session 1 (suggesting that subjects switched attention between dimensions as they did in Experiment 1); but they were smaller than the neutral-cue standard deviations from Session 8. These observations were supported by Fisher's LSD test: Neutral-cue

Table 3

Standard Deviations as a Function of Days, Compatibility, and Cue Delay in Experiment 3

\begin{tabular}{|c|c|c|c|c|c|c|}
\hline \multirow[b]{2}{*}{ Day } & \multicolumn{6}{|c|}{ Cue delay (msec) } \\
\hline & 100 & 200 & 400 & 600 & 800 & 1,000 \\
\hline \\
\hline Comp & 111 & 89 & 81 & 102 & 105 & 102 \\
\hline $2^{\text {Conf }}$ & 98 & 85 & 99 & 92 & 100 & 82 \\
\hline Comp & 143 & 126 & 127 & 116 & 107 & 133 \\
\hline${ }_{3}^{\text {Conf }}$ & 139 & 119 & 139 & 107 & 96 & 162 \\
\hline Comp & 136 & 168 & 121 & 1.48 & 104 & 124 \\
\hline Conf & 121 & 145 & 123 & 145 & 114 & 117 \\
\hline 4 & & & & & & \\
\hline Comp & 180 & 150 & 143 & 137 & 128 & 160 \\
\hline${ }_{5}^{\text {Conf }}$ & 150 & 144 & 140 & 114 & 140 & 133 \\
\hline Comp & 126 & 136 & 126 & 116 & 124 & 123 \\
\hline $6^{\text {Conf }}$ & 136 & 147 & 118 & 129 & 123 & 133 \\
\hline Comp & 129 & 115 & 131 & 139 & 131 & 138 \\
\hline Conf & 123 & 129 & 138 & 121 & 125 & 124 \\
\hline 7 & & & & & & \\
\hline Comp & 124 & 113 & 110 & 131 & 147 & 144 \\
\hline${ }_{8}^{\text {Conf }}$ & 123 & 119 & 162 & 114 & 142 & 124 \\
\hline Comp & 136 & 136 & 164 & 129 & 144 & 145 \\
\hline Conf & 173 & 181 & 197 & 145 & 152 & 170 \\
\hline
\end{tabular}

Note. Comp $=$ compatible trials; Conf $=$ conflicting trials; the cue was neutral on Days 1 and 8.
Table 4

Proportion of Errors as a Function of Days, Compatibility, and Cue Delay in Experiment 3

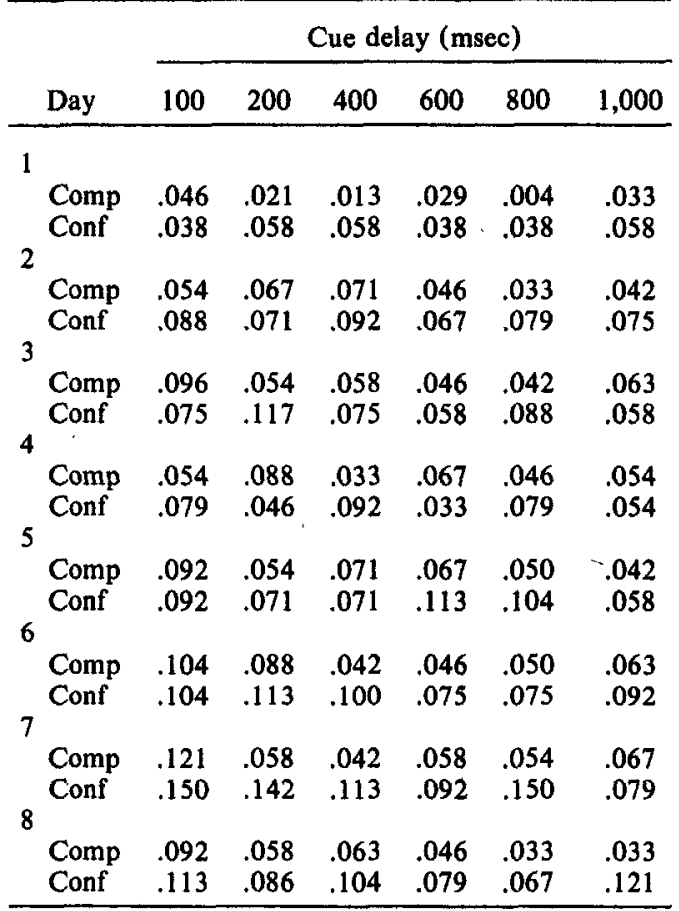

Note. Comp $=$ compatible trials; Conf $=$ conflicting trials; the cue was neutral on Days 1 and 8.

standard deviations from Session 1 were significantly smaller than the average of the cue-valid standard deviations from Sessions 2-7 and significantly smaller than the neutral-cue standard deviations from Session 8 $\left(p<.05, M S_{\mathrm{c}}=10,725.29\right)$. No effects in the ANOVA on the standard deviations were significant.

The mean error rates across subjects are presented in Table 4. Error rates tended to be higher with conflicting stimuli than with compatible ones, and higher with the cue valid than with the cue neutral.

\section{Discussion}

There were three principal findings in Experiment 3. The first was that the cue validity effect replicated once again. Again, there was some evidence that subjects switched attention between dimensions to produce the cuing effect; cued standard deviations were larger than the neutral-cue standard devia- 
tions from Session 1 and remained stable throughout practice, as did the asymptotic cued reaction times. It was puzzling to find that neutral-cue standard deviations increased on Session 8 after six sessions' practice with the cues. One possibility is that the construction process became automatized somewhat over practice, so that the presentation of an X produced an expectation for compatible stimuli and the presentation of an $O$ produced an expectation for conflicting stimuli. The former expectation would facilitate performance when compatible stimuli appeared and inhibit performance when conflicting stimuli appeared, whereas the latter expectation would do the opposite. Since the cue was neutral on Session 8, the $\mathrm{X}$ was followed by compatible stimuli and conflicting stimuli equally often, and the same was true of the $O$. Thus, the automatic effects would cancel each other out in terms of mean reaction time but would inflate the standard deviations. The obvious test of this hypothesis would be to compare compatible and conflicting reaction times following an $\mathrm{X}$ with those following an $O$. Unfortunately, we have no record of which cue preceded which stimulus, as we did not anticipate this possibility.

An alternative interpretation is that with practice, subjects learned to divide attention between dimensions, so that in the end, their cued standard deviations were smaller than neutral-cue control standard deviations. This interpretation assumes that cued standard deviations diminished with practice and that neutral-cue standard deviations were no larger on Day 8 than they were on Day 1; both of these assumptions were falsified by the data (see Table 3).

The second major result was the effect of practice on the time-course of cuing: With practice, the cue validity effect reached asymptote sooner, but the asymptotic level remained the same. This suggests that practice increased the speed of construction, which stands in marked contrast to the effects of the informational constraint manipulated in Experiments 1 and 2. That manipulation increased the cue validity effect at all cue delays without affecting the rate at which it reached asymptote, suggesting an increase in the probability of construction.
The third major finding was the advantage for cuing compatible stimuli, which remained stable over six sessions of practice. The effect replicates Experiments 1 and 2; its persistence over the six sessions of practice suggests that it may reflect some permanent, structural limitation on processing multidimensional stimuli. Since practice usually has its strongest effects early (i.e., the rate of improvement with practice is negatively accelerated), it seems unlikely that further practice would reduce the advantage.

\section{Experiment 4}

In Experiments 1, 2, and 3, the delay between the onset of the warning signal and the onset of the cue varied inversely with the delay between the onset of the cue and the onset of the word (i.e., cue delay); the two deláys always summed to $1,000 \mathrm{msec}$. Thus, it is possible that the effects we have attributed to cue delay are really due to the delay between the warning signal and the onset of the cue. Possibly, performance improved as cue delay increased because there was less time between the warning signal and the cue to adopt inefficient or maladaptive strategies. Experiment 4 was designed to remove the confound between warning interval and cue delay. Three levels of cue delay (200, 500 , and $800 \mathrm{msec}$ ) were combined factorially with three levels of warning interval $(200,500$, and $800 \mathrm{msec})$ to assess their independent and interactive effects on the time-course of the cue validity effect (note that this design includes three conditions in which warning interval and cue delay sum to $1,000 \mathrm{msec}$, exactly replicating the previous experiments). In all other respects, Experiment 4 was a replication of Experiment 1 (i.e., the two cues were valid in the same blocks, and subjects had one session with the cue valid and one session with the cue neutral).

\section{Method}

Subjects. Eighteen senior undergraduates, graduate students, and laboratory staff from Erindale College of the University of Toronto were paid for serving in two 1-hour sessions.

Apparatus and stimuli. These were the same as in the previous experiments, except that the response buttons were replaced by a set of telegraph keys that re- 


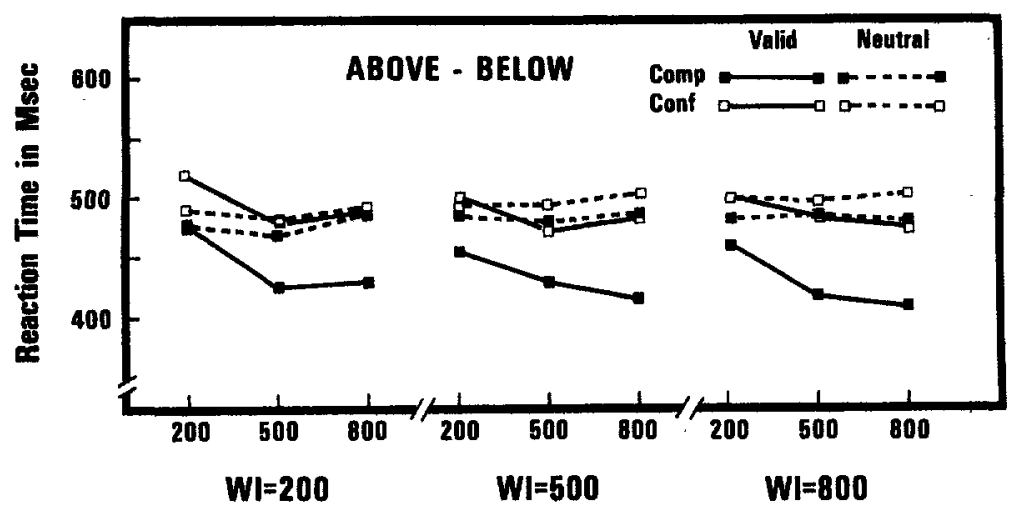

Figure 4. Mean reaction time as a function of cue delay and warning interval in Experiment 4. (Compatibility and cue validity are parameters. Comp $=$ compatible trials; Conf $=$ conflicting trials; Valid $=$ perfect cue validity; Neutral $=$ no cue validity; $W I=$ warning interval.)

quired a smaller and less forceful movement to register a response.

Procedure. Each subject completed nine blocks of 80 trials each day for two days. The nine blocks each day represented the factorial combination of three warning intervals $(200,500$, and $800 \mathrm{msec})$ and three cue delays $(200,500$, and $800 \mathrm{msec})$. Each day the order of the nine blocks varied between subjects according to two nested $3 \times 3$ Latin squares, in which cue delay varied faster than warning interval (i.e., subjects completed one block at each cue delay before moving on to the next warning interval). This created nine orders of conditions, and two subjects were assigned to each order.

Cue validity was manipulated as in Experiments 1 and 3 (i.e., both cues were valid in the same blocks; the cue either had perfect validity or none at all; neutralcue controls were used). One of the subjects assigned to each of the nine orders of conditions had cue-valid trials in the first session and neutral-cue trials in the second, whereas the other subject had the opposite.

In all other respects, the experiment was a replication of Experiment 1.

\section{Results}

Each subject completed 40 trials in each combination of the two compatibility conditions, the two cue validity conditions, the three cue delays, and the three warning intervals. Error rates and the mean reaction times and standard deviations were calculated for each subject in each condition. The mean reaction times across subjects are displayed in Figure 4. (Each point in the figure is based on a maximum of 720 observations.)

The figure shows that the pattern observed in the previous experiments replicated at each warning interval: The cuing effect was present and increased with cue delay, con- flicting stimuli were generally responded to more slowly than compatible stimuli, and the cue validity effect was much stronger with compatible stimuli than with conflicting stimuli. By contrast, warning interval had a negligible effect on performance.

These effects were apparent in a $2 \times 2 \times$ $3 \times 3$ ANOVA on the mean reaction times. The cuing effect was apparent as a significant main effect of cue validity, $F(1,17)=$ 5.37, $p<.01, M S_{e}=19,752.38$, a significant main effect of cue delay, $F(2,34)=17.49$, $p<.01, M S_{e}=1,387.50$, and a significant interaction between cue validity and cue delay, $F(2,34)=21.17, \quad p<.01, M S_{\mathrm{e}}=$. $1,216.86$. The slower responses to conflicting stimuli than to compatible ones produced a significant main effect of compatibility, $F(1$, $17=70.76, p<.01 . M S_{\mathrm{c}}=2,631.70$. A contrast comparing compatible and conflicting reaction times in the neutral-cue trials was also significant, $F(1,17)=11.36, p<.01$, $M S_{\mathrm{e}}=1,556.97$, indicating a Stroop-type effect. The finding that the cuing effect was stronger with compatible stimuli than with conflicting stimuli was evident as a significant interaction between cue validity and compatibility, $F(1,11)=34.48, p<.01$, $M S_{\mathrm{e}}=1,556,96$, a marginally significant interaction between cue validity, compatibility, and cue delay, $F(2,34)=3.12, p<.06$, $M S_{\mathrm{e}}=416.87$, and a significant interaction between compatibility and cue delay, $F(2$, $34)=3.38, p<.05, M S_{\mathrm{e}}=485.94$.

By contrast, the main effect of warning 
Table 5

Standard Deviations as a Function of Cue Validity, Warning Interval, Compatibility, and Cue Delay in Experiment 4

\begin{tabular}{|c|c|c|c|}
\hline \multirow{2}{*}{$\begin{array}{l}\text { Warning } \\
\text { interval }\end{array}$} & \multicolumn{3}{|c|}{ Cue delay (msec) } \\
\hline & 200 & 500 & 800 \\
\hline \multicolumn{4}{|c|}{ Valid cue } \\
\hline \multicolumn{4}{|l|}{200} \\
\hline Comp & 92 & 95 & 99 \\
\hline Conf & 96 & 91 & 107 \\
\hline \multicolumn{4}{|l|}{500} \\
\hline Comp & 82 & 95 & 94 \\
\hline Conf & 82 & 89 & 98 \\
\hline \multicolumn{4}{|l|}{800} \\
\hline Comp & 87 & 91 & 95 \\
\hline Conf & 85 & 88 & 86 \\
\hline \multicolumn{4}{|c|}{ Neutral cue } \\
\hline \multicolumn{4}{|l|}{200} \\
\hline Comp & 84 & 84 & 83 \\
\hline 500 & 78 & 89 & 73 \\
\hline Comp & 99 & 84 & 103 \\
\hline Conf & 81 & 86 & 89 \\
\hline \multicolumn{4}{|l|}{800} \\
\hline $\begin{array}{l}\text { Comp } \\
\text { Conf }\end{array}$ & $\begin{array}{l}83 \\
81\end{array}$ & $\begin{array}{l}85 \\
81\end{array}$ & $\begin{array}{l}90 \\
77\end{array}$ \\
\hline & 01 & 01 & \\
\hline
\end{tabular}

Note. Comp $=$ compatible trials; Conf $=$ conflicting trials.

interval was not significant, $F(2,34)<1$, $M S_{\mathrm{e}}=2,067.25$. The only significant effect involving warning interval was the interaction between warning interval and cue validity, $F(2,34)=4.87, p<.05, M S_{\mathrm{e}}=$ $1,589.39$, indicating a weaker cue validity effect at the $200-\mathrm{msec}$ warning interval (13 msec) than at the 500- and 800-msec warning intervals ( 32 and $34 \mathrm{msec}$, respectively).

The mean standard deviations across subjects are displayed in Table 5. As in the previous experiments, cue-valid standard deviations tended to be larger than cue-neutral ones ( 92 vs. $85 \mathrm{msec}$ ); indeed, cued standard deviations were larger than neutral ones in 16 of the 18 cases in Table 5. However, the difference was not significant, $F(1,11)=$ $2.10, p<.20, M S_{e}=3,565.12$, possibly because the error term was so large (it was the largest in the analysis, more than twice as large as the second largest). The only significant effect in an ANOVA on the standard deviation data was the main effect of compatibility, $F(1,11)=5.17, p<.05, M S_{\mathrm{e}}=$ 453.45. Note that the main effect of warning interval was not significant, $F(2,34)=1.19$, $M S_{e}=890.80$, nor were any of its interactions with other variables.

The mean error rates across subjects are displayed in Table 6. As in the previous experiments, subjects made more errors when the cue was valid than when the cue was neutral; they also made more errors with conflicting stimuli than with compatible stimuli. Further, the difference between compatible and conflicting stimuli was magnified when the cue was valid. This pattern appeared stable across warning intervals and cue delays.

\section{Discussion}

This experiment confirmed the major findings of the previous experiments with a design in which cue delay was not confounded with warning interval. In fact, warning interval had very little effect on performance. The only significant effect involving warning interval was the interaction with cue validity, in which the cue validity effect was stronger at the 500- and 800-msec warning intervals than at the $200-\mathrm{msec}$ warning interval. At worst, this suggests that the previous experiments may have underestimated the cue validity effect at the 800 - and 1,000 msec cue delays, which had warning intervals of $200 \mathrm{msec}$ or less. However, they would not have been underestimated by much because the warning interval effect in the present experiment was relatively weak. Note that warning interval should have no effect on estimates of the latency of construction, which have been based primarily on data from the 100-, 200-, 400-, and 600msec cue delays with warning intervals ranging from 400 to $900 \mathrm{msec}$.

Note as well that reaction times in this experiment were faster overall than reaction times in the previous experiments. This was probably because the telegraph keys in the present experiment were easier to press than the buttons in the previous experiments. Apparently, whatever was responsible for the increase in speed did not interact with 
the major variables of interest, since the pattern of effects was the same as in the previous experiments.

\section{General Discussion}

The present experiments were successful in inducing subjects to construct strategies, which utilized information about the relation between stimulus dimensions, in order to maximize the goal of reporting the identity of the word as quickly as possible. The timecourse of constructing these strategies was found to be sensitive to constraints imposed by the informational structure of the task environment. It was also affected by the subject's repertoire of cognitive processes. Certain effects were found to be invariant over changes in strategy; there were related to the Stroop-like nature of the stimuli. Each is discussed in turn.

\section{Cue Validity Effects}

The clearest result in all four experiments was the cue validity effect: The presence of a cue reduced reaction time below the level of neutral-cue or no-cue controls in each experiment. This replicates the strategic use of relations between dimensions seen in the earlier studies (Greenwald \& Rosenberg, 1978; Logan, 1980; Logan \& Zbrodoff, 1979). The present studies go beyond the earlier ones in demonstrating cue validity effects without a confound between cue validity and stimulus frequency; in the earlier studies, the stimuli that benefited most from cuing were the most frequent, whereas in the present studies (except Experiment 2), all stimuli were equally frequent.

It is interesting that the cuing effect in the present experiments may have resulted from a different strategy than the cuing effect seen in the earlier studies. The inflated standard deviations of the cued reaction times in the present experiments suggest that subjects were switching attention between dimensions, basing their responses on the word's position on some trials, and basing their responses on the word's identity on the others. By contrast, it is clear that subjects in the earlier studies divided attention between di-
Table 6

Proportion of Errors as a Function of Warning Interval, Cue Validity, Compatibility, and Cue Delay in Experiment 4

\begin{tabular}{|c|c|c|c|}
\hline \multirow{2}{*}{$\begin{array}{l}\text { Warning } \\
\text { interval }\end{array}$} & \multicolumn{3}{|c|}{ Cue delay (msec) } \\
\hline & 200 & 500 & 800 \\
\hline \multicolumn{4}{|c|}{ Valid cue } \\
\hline \multicolumn{4}{|l|}{200} \\
\hline Comp & .035 & .042 & .028 \\
\hline Conf & .053 & .047 & .051 \\
\hline \multicolumn{4}{|l|}{500} \\
\hline Comp & .047 & -.028 & .024 \\
\hline Conf & .071 & .061 & .051 \\
\hline \multicolumn{4}{|l|}{800} \\
\hline Comp & .032 & .018 & .021 \\
\hline Conf & .060 & .069 & .060 \\
\hline \multicolumn{4}{|c|}{ Neutral cue } \\
\hline \multicolumn{4}{|l|}{200} \\
\hline Comp & .031 & .029 & .031 \\
\hline Conf & .046 & .017 & .029 \\
\hline \multicolumn{4}{|l|}{500} \\
\hline Comp & .026 & .022 & .024 \\
\hline Conf & .021 & .033 & .017 \\
\hline \multicolumn{4}{|l|}{800} \\
\hline Comp & .025 & .019 & .028 \\
\hline Conf & .038 & .028 & .025 \\
\hline
\end{tabular}

Note. Comp $=$ compatible; Conf $=$ conlicting trials.

mensions, basing their responses partly on the word's position and partly on its identity: In those experiments, the cue was not perfectly valid, so subjects who attended only to the word's position would make errors each time they encountered a stimulus that was inconsistent with their expectation (e.g., if conflicting stimuli were frequent, subjects could respond "above". when the word appeared below the fixation point and "below" when it appeared above; this would result in fast, accurate responses to conflicting stimuli but would produce errors with every compatible stimulus). However, the error rates were far too low for subjects to have switched attention to position often enough to have produced the observed cue validity effects.

This contrast between the earlier studies and the present ones is more likely a consequence of differences in cue validity than of differences in the method of cuing (i.e., cuing with a precue versus manipulating the relative frequency of compatible and conflicting trials). In order to maximize the goal 
of responding accurately, subjects should attend exclusively to the word's position only when cue validity is high enough that unexpected stimuli are relatively rare (i.e., rare enough that the errors they produce will not inflate the overall error rate beyond acceptable limits). Given the usual instruction to keep error rate below $10 \%$, subjects should not attend exclusively to position unless cue validity is in excess of $90 \%$. In the present studies that showed evidence of selective attention, cue validity was $100 \%$; in the earlier studies that showed evidence of divided attention, cue validity ranged from $60 \%$ to $90 \%$. Thus, the differences in strategy (selective vs. divided attention) may be manifestations of a general "metastrategy" of attending to the word's position in proportion to its validity as a cue to the identity of the word (see Logan \& Zbrodoff, 1979).

There was also a cue validity effect in the error data of each experiment: Presenting a cue increased error rates relative to neutralcue or no-cue controls, suggesting (empirically at least) a speed-accuracy trade-off. Indeed, other studies of cuing have found such trade-offs (e.g., LaBerge, 1973), and interpretations of cuing in terms of adjusting a temporal or evidential criterion (e.g., Murrell, 1977) would predict their occurrence (but see Antos, 1979). We suggest that the increased error rate might be a consequence of dealing with the cue rather than simply adjusting a temporal or evidential criterion. That is, the response to the cue may have engaged some extra, error-prone processes that were not required in the no-cue or neutral-cue conditions, and this may have produced the increase in error rate. Subjects' reports that it was difficult and sometimes confusing to use the cue would support this interpretation.

\section{Constraints on Strategy Construction and Utilization}

A major purpose of the present research was to determine how subjects' strategies changed in response to changes in the constraints imposed by the informational structure of the task environment and by the subject's cognitive abilities. The different constraints affected strategy construction in different ways: Practice affected the rate at which the cue validity effect reached asymptote but had little effect on the asymptote itself. By contrast, varying the number of potential cues that could occur in a block affected the asymptote and had little effect on the rate of approach to the asymptote. According to the logic developed in the introduction, the former, subject-related, constraint affected the speed of construction but had no effect on the probability of construction or the efficiency of utilization. Further, the latter, informational, constraint affected the probability of construction but had no effect on the speed of construction or the efficiency of utilization.

It is interesting that the probability of constructing a strategy was independent of the speed of construction. One would expect that subjects might come to rely more on a strategy as it becomes more efficient. However, both cues were valid throughout practice, and it is important to remember that they signaled opposite responses; this conflict may have made construction so difficult that the improvement in speed was small compensation for the effort involved. A more detailed study of the trade-off function would be necessary to test this hypothesis adequately (see Navon \& Gopher, 1979).

It would be interesting to see whether strategy construction and utilization respond similarly to similar constraints in other paradigms. Given the common idea that strategies are temporary options exercised on a moment's notice, construction is a necessary precursor to utilization. Very generally, construction is a special case of interpreting instructions (i.e., compiling the symbolic content of the instructions into a procedure for performing the task), and utilization is a special case of behaving in accord with instructions (i.e., executing the compiled procedure to fulfill the instructed goals). Clearly, strategy construction and utilization are very important in everyday cognition, and it is important that psychologists come to understand them. The task is difficult, however, because strategies are inherently flexible and versatile, and so appear to resist static description. Perhaps the major contribution of 
the present experiments is to show that subjects vary their strategies systematically in response to constraints that can be manipulated experimentally. This suggests a paradigm for studying strategies in which one describes the range of strategies possible within the constraints imposed by the goal structure, the subject's abilities, and the structure of the task environment, and discovers the way(s) in which the strategies respond to variations in the constraints.

\section{Stroop-Like Effects}

In each experiment, the compatibility of the word and the position it occupied affected performance. There were three distinct effects. First, in the neutral-cue trials of Experiments 1, 3, and 4, reaction times to conflicting stimuli were longer than reaction times to compatible stimuli. This compatibility effect reflects (the sum of) Stroop-like facilitation and inhibition, replicating previous findings (Logan, 1980; Logan \& Zbrodoff, 1979; Palef, 1978; Palef \& Olson, 1975).

The second effect was the variation in Stroop-like facilitation and interference in the no-cue trials of Experiment 2. In that experiment, the magnitude of the no-cue Stroop effect depended on the cue that was valid at the time. Cuing compatible stimuli increased the difference between compatible and conflicting reaction times in the no-cue trials, whereas cuing conflicting stimuli reduced it. This may be interpreted as a carryover effect of having used the cue, since the no-cue stimuli that benefited from the carryover were those that were consistent with the relation that the cue signaled, and the nocue stimuli that suffered were those that conflicted with the relation signaled by the cue. This interpretation is important because it suggests that attending to the unreported dimension even occasionally may bias Strooptype interference on subsequent trials, which suggests that many Stroop-type effects may be a consequence of occasional attention to the unreported dimension.

Alternatively, the effect in the no-cue trials may depend on stimulus frequency, quite independent of the cue. Indeed, the data are very similar quantitatively to data from our earlier experiments (Logan \& Zbrodoff, 1979) in which no cues were presented. In the present Experiment 2, conflicting stimuli occurred on $33 \%$ of the trials in blocks in which $X$ was the cue, and the compatibility effect was $67 \mathrm{msec}$; in our earlier experiment, when conflicting stimuli occurred on $40 \%$ of the trials, the compatibility effect was $62 \mathrm{msec}$ (Logan \& Zbrodoff, 1979, Experiment 2). In the present Experiment 2 , conflicting stimuli occurred on $66 \%$ of the trials when $O$ was the cue, and the compatibility effect was $-7 \mathrm{msec}$; in our earlier experiment, when conflicting stimuli occurred on $60 \%$ of the trials, the compatibility effect was 5 msec (Logan \& Zbrodoff, 1979, Experiment 2). However, this may be no more than coincidence; further research must be done to determine whether the variation in the no-cue compatibility effect resulted from having used the cue or from stimulus frequency alone.

The third Stroop-like effect was that cuing produced stronger effects with compatible stimuli than with conflicting ones. This occurred in all four experiments, and it was not reduced by practice or by reducing the number of potential cues that could occur in a block. This resembles an asymmetry seen in the cue validity effect produced by varying the relative frequency of conflicting trials. In those studies, the difference in reaction time to expected and unexpected stimuli was greater when compatible stimuli were expected than when conflicting stimuli were expected. Those asymmetries could be accounted for by assuming that the Strooplike effects and the cue validity effects were additive and thus complemented each other when compatible stimuli were expected and counteracted each other when conflicting stimuli were expected. The asymmetries in the present experiments cannot be explained by an additive model; the cue validity effects were stronger with compatible stimuli than with conflicting ones, even when the Strooptype effect was subtracted out (i.e., by assessing the cue validity effect for compatible trials against the neutral-cue or no-cue compatible reaction times, and assessing the cue validity effect for conflicting trials against 
neutral-cue or no-cue conflicting reaction times).

Perhaps this difference was a consequence of dividing attention in the earlier experiments and switching attention in the present ones. In the present studies, subjects may have switched attention to the word's position more often when compatible stimuli were cued than when conflicting stimuli were cued, whereas in the earlier studies, subjects may have divided attention as often and to the same extent when compatible trials were frequent as when conflicting trials were frequent. However, the lack of effects of practice and reducing the number of potential cues that could occur in a block suggests that the difference in cue validity effects might reflect structural, strategy-invariant limitations on construction (i.e., constraints imposed by the structure of the subject's cognitive abilities), rather than strategic limitations as the interpretation in terms of differences in the probability of construction suggests.

An alternative interpretation comes to mind when the interaction between cue validity and compatibility is described differently; the compatibility effect was much stronger when the cue was valid than when it was neutral. This suggests that the strategic use of position information amplifies the automatic compatibility effects, as if attention to the word's position activated habitual associations between word identity and position more than they would have been activated had attention been restricted to word identity. Similarly, Kahneman and Henik (1980) and Francolini and Egeth (1980) suggested that attention to objects in space might amplify the automatic effects associated with them. Kahneman and Henik (1980) presented two words, one black and one colored, one of which named a conflicting color and one of which was neutral. The subject's task was to name the color of the colored word. Interference from the conflicting color word was much stronger when the conflicting color word was colored and the neutral word was black than when the conflicting color word was black and the neutral word was colored. Francolini and Egeth (1980) had subjects count the red characters in arrays of red and black letters and digits; they found that the interference produced by an irrelevant digit (e.g., 3333) was much stronger if the digit was red (hence attended) than if it was black (hence unattended).

The idea that automatic effects may be amplified by attention is important and is worth pursuing in further research. The present procedure may prove valuable in this respect because there is an alternative explanation for the compatibility effect being larger with a cue than without (i.e., the probability of construction may differ). The procedures of Kahneman and Henik (1980) and Francolini and Egeth (1980) do not provide such a clear and distinguishable alternative.

\section{Conclusions}

The present experiments have demonstrated strategic use of the relation between the identity of a word and the position it occupied in the viewing screen, corroborating previous experiments using a different method of cuing the relation (Greenwald \& Rosenberg, 1978; Logan, 1980; Logan \& Zbrodoff, 1979). Whereas the previous experiments focused primarily on strategy utilization in response to informational constraints, the present experiments focused on strategy construction and showed that the construction process was sensitive to manipulations of informational and subject-related constraints. In particular, informational constraints affected the probability of construction (Experiments 1 and 2), and subject-related constraints (practice) affected the speed of construction (Experiment 3). Perhaps the most important conclusion to be drawn from these experiments is that strategies behave systematically and are therefore susceptible to experimental investigation.

\section{References}

Antos, S. J. Processing facilitation in a lexical decision task. Journal of Experimental Psychology: Human Perception and Performance, 1979, 5, 527-545.

Dyer, F. N. The Stroop phenomenon and its use in the study of perceptual, cognitive and response factors. Memory \& Cognition, 1973, I, 106-120.

Francolini, C. M., \& Egeth, H. On the nonautomaticity of "automatic" activation: Evidence of selective seeing. Perception \& Psychophysics, 1980, 27, 331-342. 
Greenwald, A. G., \& Rosenberg, K. E. Sequential effects of distracting stimuli in a selective attention reaction time task. In J. Requin (Ed.), Attention and performance VII. Hillsdale, N.J.: Erlbaum, 1978.

Jensen, A. R., \& Rohwer, W. D. The Stroop color-word test: A review. Acta Psychologica, 1966, 25, 36-93.

Kahneman, D., \& Henik, A. Perceptual organization and attention. In M. Kubovy \& J. Pomerantz (Eds.), Perceptual organization. Hillsdale, N.J.: Erlbaum, 1980.

Kirk, R. E. Experimental design: Procedures for the behavioral sciences. Monterey, Calif.: Brooks/Cole, 1968.

LaBerge, D. Identification of two components of the time to switch attention: $A$ test of a serial and a parallel model of attention. In S. Kornblum (Ed.), Attention and performance IV. New York: Academic Press, 1973.

Logan, G. D. Attention and automaticity in Stroop and priming tasks: Theory and data. Cognitive Psychology, 1980, 12, 523-553.

Logan, G. D., \& Zbrodoff, N. J. When it helps to be misled: Facilitative effects of increasing the frequency of conflicting trials in a Stroop-like task. Memory \& Cognition, 1979, 7, 166-174.

Murrell, G. A. Combination of evidence in a probabilistic visual search and detection task. Organizational Behavior and Human Performance, 1977, 18, 3-18.

Navon, D., \& Gopher, D. On the economy of the human processing system. Psychological Review, 1979, 86, 214-255.

Palef, S. R. Judging pictorial and linguistic aspects of space. Memory \& Cognition, 1978, 6, 70-75.

Palef, S. R., \& Olson, D. R. Spatial and verbal rivalry in a Stroop-like task. Canadian Journal of Psychology, 1975, 29, 201-209.

Posner, M. I., \& Boies, S. J. Components of attention. Psychological Review, 1971, 78, 391-408.

Ratcliff, R. A theory of memory retrieval. Psychological Review, 1978, 85, 59-108.

Received June 22, 1981

Revision received October 7, 1981 